\title{
An Integrated Deep Learning Framework of Tomato Leaf Disease Detection
}

\author{
Jiten Khurana, Anurag Sharma, Harshit Singh Chhabra, Rahul Nijhawan
}

\begin{abstract}
Plants are a very important part of human life. They have variety of use as food, medication, raw materials and maintaining a balanced ecosystem. Plant disease is a deterioration of normal state of plant that interrupts and modifies its functionality. Pathogens are the main cause of such diseases. For agricultural purposes, a variety of methods have been proposed to detect plant diseases in the recent technological era. However, detecting plant diseases with high accuracy is still a challenge in computer vision. In this study, we propose an integrated deep learning framework where a pre-trained VGG-19 model is used for feature extraction and stacking ensemble model is used to detect and classify leaf diseases from images so as to reduce production and economic loses in agriculture sector. A dataset consisting of two classes (Infected and Healthy) and a total of 3242 images was used to test the system. Our proposed work has been compared with other contemporary algorithms ( $k N N, S V M, R F$ and Tree) and have outperformed by obtaining an accuracy of $98.6 \%$.
\end{abstract}

Keywords: Disease Classification, Deep Learning Technique, Hybrid, Bacterial Spots, Leaf Curl.

\section{INTRODUCTION}

Disease in plants can be caused by several factors which can result in economical and production loss. Climate change is one of the most important factor that decide the fortune of the plants. There are several pathogens that can cause tomato plant disease. Pathogens can be insects or micro-organisms like viruses and bacteria. The rate of infection in tomato plants are dependent of factors like wind pattern, temperature, humidity, varietal resistance and plant's health. Disease like leaf bacterial spot is caused in moist weather and splattering rains, leaf curl disease is characterized by stunting of the plants with downward rolling and crinkling of the leaves, mosaic which is characterized by light and dark green spot on leaves.

Detection of infected leaves is a relatively fresh field in which there is lot of potential for research. Alvaro Fuentes et al [1] have used imaging and spectroscopic techniques on several images to generate characteristics of tomato plant diseases. Rajvinder Kaur et al [2] have used k- Nearest Neighbor (kNN), Neural Network, SVM model on several images of unhealthy plant leaves and got an accuracy of $91 \%$ in testing to classify the leaf images. Bin Liu et al [3]

Revised Manuscript Received on September 10, 2019.

Jiten Khurana, Graphic Era University, Dehradun (U.K.), India

Anurag Sharma, Graphic Era University, Dehradun (U.K.), India

(Email: anurag.sharma.kaku@gmail.com) India

(Email: harshitchhabra8@gmail.com)

Rahul Nijhawan, Graphic Era University, Dehradun (U.K.), India

(Email: dr.rahul.iitr@gmail.com)
(Email: jitenkhurana131@gmail.com)

have used Deep Convolutional Neural Networks (CNN), Random Forest (RF), K-Nearest Neighbor (kNN) and SVM models on a dataset of 13,689 images of disease apple leaves and got an accuracy of $97.62 \%$ using CNN model. Muhammad Majid Sharif et al [4] have done work on detection and classification of citrus diseases in crops using optimized weighted segmentation and feature selection. They have used Multiclass Support Vector Machine (MSVM) on three datasets - citrus diseases dataset having 670 images, local dataset with 580 images and combined dataset with 5,632 images and got an accuracy of $97 \%, 90.4 \%$ and $89 \%$ respectively. Compared to all of these our proposed work uses ensemble model on dataset of 3,242 images of tomato leaf diseases and disorders to get an accuracy of $98.6 \%$.

We faced several challenges during our work. The biggest challenge that we have faced was the lack of an existing appropriate dataset. To overcome this problem we had to modify the existing datasets using several resources to meet our needs. Another problem which we faced was some plant leaves looked similar while others had multiple diseases.

In our paper an integrated deep learning approach for detection of tomato leaf diseases and disorders is proposed. We have used pre-trained VGG19 model [5] which is a 19 layers deep model trained on ImageNet to get the feature vector of the images. After that we have employed a combination of different stacking models to get the results. Our proposed model outperformed the contemporary algorithms like kNN, SVM, RF and Decision Tree. Further accuracy assessment has been performed using statistical measures (Accuracy, Specificity, Sensitivity, F1 score and AUC).

This paper is divided in the following order: I. Introduction, II. Data Collection, III. Methodology, IV. Proposed approach, V. Results and Discussions, VI. Conclusion.

\section{DATA COLLECTION}

Data collection plays an important role in solving machine learning problems and hence it is a big challenge in this field. We have built our dataset by collecting various images from existing open source datasets dealing with tomato leaf disease. The final dataset consisting of two classes of tomato leaf having 3242 images. Table I shows the distribution of images in our dataset.

Published By: 
TABLE I

DISTRIBUTION OF IMAGES IN THE DATASET

\begin{tabular}{|c|c|}
\hline Tomato Leaf Class & Number of images \\
\hline Infected & 1651 \\
\hline Healthy & 1591 \\
\hline Total & 3242 \\
\hline
\end{tabular}

Our dataset was randomly split into two parts where $85 \%$ of data was used for training while the rest was used for testing our models.

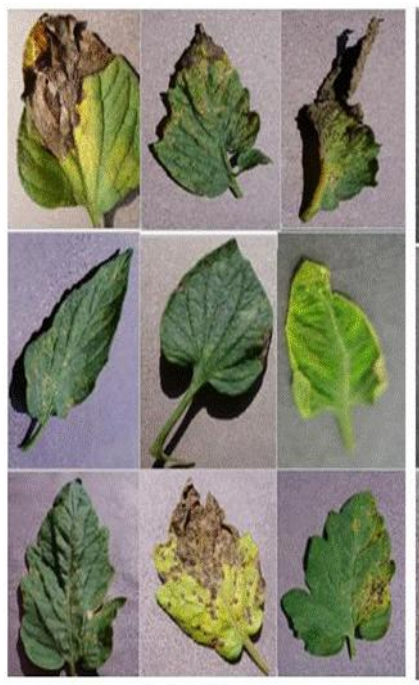

Infected Leaves

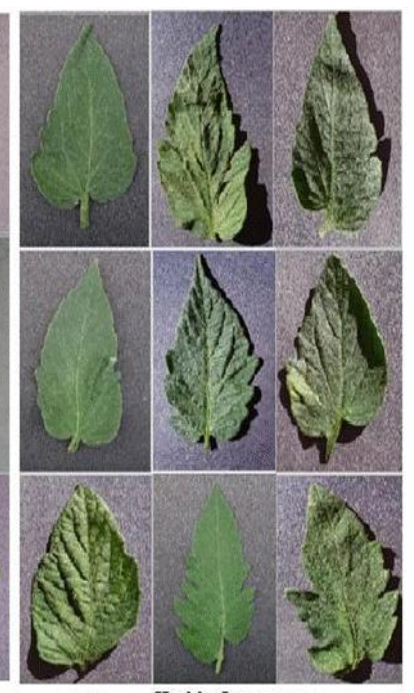

Healthy Leaves
Figure 1. Sample of images in our dataset

\section{METHODOLOGY}

In this study we have used stacking or stacked generalization to perform classification on our dataset. We have implemented our research on a machine with quadcore Intel I5 processor with 8GB RAM and 2GB (AMD) GPU.

First we have used pre-trained VGG19 model [5] on our data-set to get features for each image. Then we ran our dataset on some state-of-the-algorithm like SVM, kNN, AdaBoost and Random Forest and compared the results to our approach of stacking.

\section{PROPOSED APPROACH}

Our study proposes a hybrid deep learning model. We have used VGG-19 model [5] in the study which has been pre-trained on ImageNet dataset for feature extraction of the dataset provided. After that stacking ensemble model is used for the classification of dataset.

The VGG-19 model [5] architecture uses only $3 * 3$ convolutional layers stacked on top of each other. This network is 19 layers deep which can classify the images into 1000 categories. Among the 19 weight layers, 11 are pure convolution layers, 5 are convolution layer and max pooling layers with two fully-connected layers which is then followed by a softmax classifier as shown in the Figure 2.

Stacking is an ensemble model for the classification and pattern recognition. This model works by combining the predictions of two or more models (base-classifiers) on the same dataset to form a new model. Stacking ensemble is most effective with the significant different base model but each model should be skillful. Our study uses Support Vector Machine, Random Forest and Artificial Neural Network as the base classifiers. The predictions are then combined using Logistic Regression to obtain the final output which is the classification of leaves as shown in Figure 2.

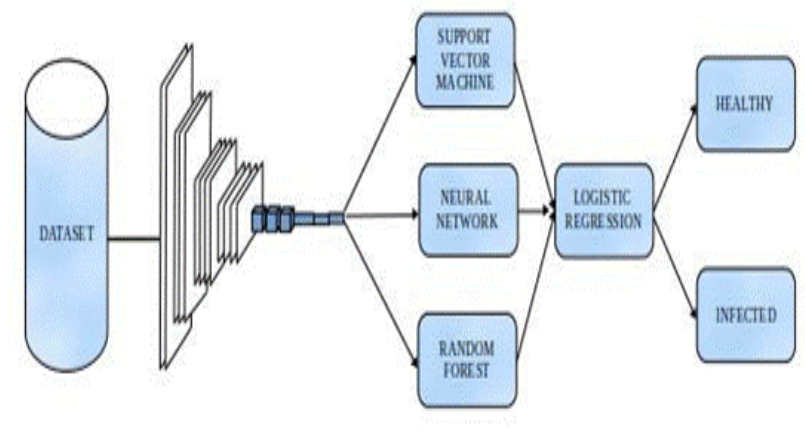

\section{FEATURE EXTRACTION CLASSIFICATION}

Figure 2. Proposed approach for Leaves Classification

\section{RESULTS AND DISCUSSION}

This section brings a series of tables and statistical plots to present the results obtained by our proposed approach and other contemporary algorithms. We have used 3 different pre-trained CNN models for feature extraction. Then we have applied various state-of-the-art algorithms along with 2 different stacking models. Model 1 uses Support Vector Machine, Random Forest and Artificial Neural Network, whereas Model 2 uses k-Nearest Neighbor, Random Forest and Decision Tree as base classifiers. Logistic Regression was used to combine the results from various classifiers in both stacking models. It was observed that the highest result (accuracy 98.6\%, F1 score 98.6\% and sensitivity 98.6\%) were obtained by our proposed approach when VGG19 model was used for feature extraction and Stacking Model 1 was used for classification. 
TABLE II ACCURACY ASSESSMENT

\begin{tabular}{|c|c|c|c|c|c|c|}
\hline $\begin{array}{l}\text { Feature } \\
\text { Extraction }\end{array}$ & Classifier & Accuracy & F1 Score & Precision & Sensitivity & AUC \\
\hline \multirow{7}{*}{ Inception v3 } & Model 1 & 98.4 & 98.4 & 98.4 & 98.4 & 99.7 \\
\hline & Model 2 & 97.1 & 97.1 & 97.1 & 97.1 & 99.4 \\
\hline & SVM[6] & 96.1 & 96.1 & 96.1 & 96.1 & 99.3 \\
\hline & $\mathrm{RF}[7]$ & 92.6 & 92.6 & 92.8 & 92.6 & 97.3 \\
\hline & kNN[8] & 89.9 & 89.9 & 88.1 & 89.9 & 95.8 \\
\hline & Tree[9] & 88.1 & 88.1 & 88.1 & 88.1 & 85.3 \\
\hline & AdaBoost[10] & 86.0 & 86.0 & 86.0 & 86.0 & 86.0 \\
\hline \multirow{7}{*}{ VGG-16 } & Model 2 & 97.7 & 97.7 & 97.7 & 97.7 & 99.8 \\
\hline & Model 1 & 97.1 & 97.1 & 97.1 & 97.1 & 99.8 \\
\hline & $\mathrm{RF}[7]$ & 95.3 & 95.3 & 95.3 & 95.3 & 98.7 \\
\hline & SVM[6] & 94.9 & 94.9 & 94.9 & 94.9 & 99.3 \\
\hline & Tree[9] & 93.2 & 93.2 & 93.2 & 93.2 & 91.5 \\
\hline & kNN[8] & 92.6 & 92.6 & 93.2 & 92.6 & 97.3 \\
\hline & AdaBoost[10] & 90.1 & 90.1 & 90.1 & 90.1 & 90.1 \\
\hline \multirow{7}{*}{ VGG-19 } & Model 1 & 98.6 & 98.6 & 98.6 & 98.6 & 99.9 \\
\hline & Model 2 & 98.4 & 98.4 & 98.4 & 98.4 & 99.8 \\
\hline & SVM[6] & 96.3 & 96.3 & 96.3 & 96.3 & 99.6 \\
\hline & $\mathrm{RF}[7]$ & 94.9 & 94.9 & 94.9 & 94.9 & 98.9 \\
\hline & kNN[8] & 92.8 & 92.8 & 93.2 & 92.8 & 96.9 \\
\hline & Tree[9] & 92.6 & 92.6 & 92.6 & 92.6 & 90.5 \\
\hline & AdaBoost[10] & 92.4 & 92.4 & 92.4 & 92.4 & 92.4 \\
\hline
\end{tabular}

We also computed ROC curves to evaluate the performance of our approach. They are made by plotting FP Rate on $\mathrm{x}$-axis along with TP rate on $\mathrm{y}$-axis. The performance of model is denoted by AUC. A model is considered perfect if AUC is close to 1 .

Generally features are manually hand-crafted which require a lot of effort. However, Deep Neural Networks [11]

[12] [13] have the ability to learn which feature are important within a very short time. They can also learn very complex functions which are very hard to understand. The activation functions in these networks also provide them the capability to exploit non-linear relations.

Stacking model is an active research area that where combining data mining models gives better result than

Published By: 
selecting each model separately. Stacking model combines specific generalization by using their output information as input into a new space instead of selecting one specific generalization out of multiple once.

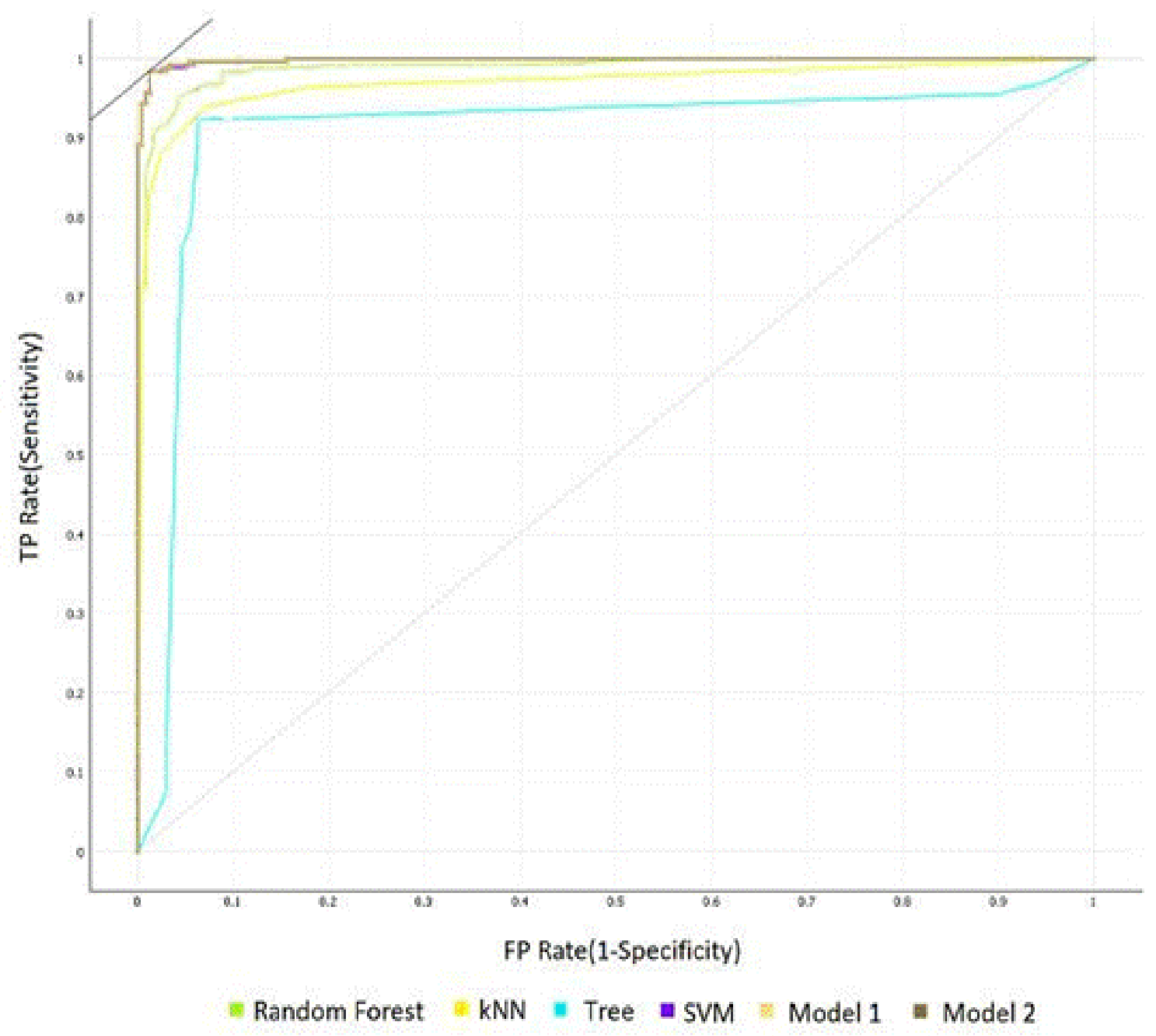

Figure 3. ROC curve for class infected (VGG19 + Model 1)

\section{CONCLUSION}

In this paper, we have proposed an integrated deep learning model which uses pre-trained VGG19 model for feature extraction and stacking ensemble model for tomato leaves classification. Taking the images for 2 different classes of tomato plant leaves, we obtained an accuracy of 98.6\%. We have shown that our approach outperformed other contemporary algorithms by the help of various statistical measures. Application for our research is to ease detection of diseases in plants which can be used to minimize the loss in the productivity of tomatoes and hence increased economic benefits. This work can easily be extended to identify infected leaves of other categories of plants. This work can also be used by agronomists to develop methods to improve the production of crops by removing the possibility of damage causing factors.

\section{REFERENCES}

1. Fuentes, A., Park, D. S., Yoon, S., Youngki, H., \& Lee, Y. Characteristics of Tomato Plant Diseases.
2. Kaur, L., \& Laxmi, V. (2016). Detection of Unhealthy Region of plant leaves using Neural Network. Dis Manag, 1(05), 34-42.

3. Liu, B., Zhang, Y., He, D., \& Li, Y. (2017). Identification of apple leaf diseases based on deep convolutional neural networks. Symmetry, 10(1), 11.

4. Sharif, M., Khan, M. A., Iqbal, Z., Azam, M. F., Lali, M. I. U., \& Javed, M. Y. (2018). Detection and classification of citrus diseases in agriculture based on optimized weighted segmentation and feature selection. Computers and electronics in agriculture, 150, 220-234.

5. Simonyan, K., \& Zisserman, A. (2014). Very deep convolutional networks for large-scale image recognition. arXiv preprint arXiv:1409.1556.

6. Ben-Hur, A., Horn, D., Siegelmann, H. T., \& Vapnik, V. (2001). Support vector clustering. Journal of machine learning research, 2(Dec), 125-137.

7. Bosch, A., Zisserman, A., \& Munoz, X. (2007, October). Image classification using random forests and ferns. In Computer Vision, 2007. ICCV 2007. IEEE 11th International Conference on (pp. 1-8). IEEE. 
8. Indriani, O. R., Kusuma, E. J., Sari, C. A., \& Rachmawanto, E. H. (2017, November). Tomatoes classification using K-NN based on GLCM and HSV color space. In 2017 International Conference on Innovative and Creative Information Technology (ICITech) (pp. 1-6). IEEE.

9. Goel, N., \& Sehgal, P. (2015). Fuzzy classification of pre-harvest tomatoes for ripeness estimation-An approach based on automatic rule learning using decision tree. Applied Soft Computing, 36, 45-56.

10. Freund, Y., Schapire, R., \& Abe, N. (1999). A short introduction to boosting. Journal-Japanese Society For Artificial Intelligence, 14(771-780), 1612.

11. Nijhawan, R., Sharma, H., Sahni, H., \& Batra, A. (2017, December). A Deep Learning Hybrid CNN Framework Approach for Vegetation Cover Mapping Using Deep Features. In 2017 13th International Conference on Signal-Image Technology \& Internet-Based Systems (SITIS) (pp. 192-196). IEEE.

12. Nijhawan, R., Verma, R., Bhushan, S., Dua, R., \& Mittal, A. (2017, December). An Integrated Deep Learning Framework Approach for Nail Disease Identification. In 2017 13th International Conference on Signal-Image Technology \& Internet-Based Systems (SITIS) (pp. 197202). IEEE.

13. Nijhawan, R., Rishi, M., Tiwari, A., \& Dua, R. (2019). A Novel Deep Learning Framework Approach for Natural Calamities Detection. In Information and Communication Technology for Competitive Strategies (pp. 561-569). Springer, Singapore. 